\title{
Novel Diagonal Reloading Based Direction of Arrival Estimation in Unknown Non-Uniform Noise
}

\author{
Hao Zhou $\mathbb{D}$, Guoping Hu $\mathbb{D}$, Junpeng Shi, and Ziang Feng \\ Air and Missile Defense College, Air Force Engineering University, Xian, China \\ Correspondence should be addressed to Guoping Hu; m15339216717@163.com
}

Received 30 January 2018; Revised 20 April 2018; Accepted 26 April 2018; Published 30 May 2018

Academic Editor: Yuri Vladimirovich Mikhlin

Copyright (C) 2018 Hao Zhou et al. This is an open access article distributed under the Creative Commons Attribution License, which permits unrestricted use, distribution, and reproduction in any medium, provided the original work is properly cited.

Nested array can expand the degrees of freedom (DOF) from difference coarray perspective, but suffering from the performance degradation of direction of arrival (DOA) estimation in unknown non-uniform noise. In this paper, a novel diagonal reloading (DR) based DOA estimation algorithm is proposed using a recently developed nested MIMO array. The elements in the main diagonal of the sample covariance matrix are eliminated; next the smallest $M N-K$ eigenvalues of the revised matrix are obtained and averaged to estimate the sum value of the signal power. Further the estimated sum value is filled into the main diagonal of the revised matrix for estimating the signal covariance matrix. In this case, the negative effect of noise is eliminated without losing the useful information of the signal matrix. Besides, the degrees of freedom are expanded obviously, resulting in the performance improvement. Several simulations are conducted to demonstrate the effectiveness of the proposed algorithm.

\section{Introduction}

Direction of arrival (DOA) estimation, which has been studied for decades, has been widely used in target localization, wireless communication, and so on. Solving the non-uniform noise problem is inevitable and important for improving the DOA estimation performance, no matter for the DOA estimation algorithm or array structure design.

For the DOA estimation algorithm, many classical methods like the Multiple Signal Classification (MUSIC), Estimation Signal Parameters via Rotational Invariance Techniques (ESPRIT), Maximum Likelihood (ML), and so on have been proposed [1]. Also, with the introduction of sparse recovery theory, the DOA estimation has come into a new era and algorithms such as Khatri-Rao MUSIC (KR-MUSIC) and Spatial Smoothing MUSIC (SS-MUSIC) are developed to improve the DOA estimation performance [2]. Most of these algorithms are based on the assumption that the received noise is uniform white Gaussian noise. However, in practical application, this assumption can hardly be guaranteed; thus the estimation performance will degrade.

For the array structure design, the structure of sensors has also been well studied to expand the degrees of freedom (DOF). Based on the subspace algorithm, traditional uniform linear arrays with $F$ elements can resolve $F-1$ targets [3]. The minimum redundancy (MR) array aims to achieve optimal structure by reducing the redundancy, but it needs complex optimization and has no closed-form expression for sensors positions $[4,5]$. The Multiple Input Multiple Output (MIMO) structure can effectively expand the DOFs by generating a virtual array [6]. But it only uses the sum co-array and there is still space to improve the DOFs. The nested array [7] and the coprime array [8] are thus proposed, which brings obvious increase of the DOFs. Taking the two level nested arrays as an example, it can obtain $O\left(F^{2}\right)$ DOF using only $O(F)$ physical sensors. The aforementioned MR array [9] and coprime array [10] are combined with the MIMO array to generate the difference co-array of the sum coarray (DCSC), which further improves the DOFs greatly. Recently, a novel structure of this type, which is called nested MIMO array, is proposed [11]. It not only expands the DOFs obviously, but also has closed-form expressions. However, the non-uniformity problem of the received noise occurs with the repaid development of these new structures. For closely spaced arrays whose inter-element spacing is less than or equal to half the wavelength, this problem is not serious and we can assume the noise obeys white Gaussian distribution. However, as the sensors of sparse arrays are usually widely 
separated, they receive noise with different power. In some special circumstance such as low altitude, the non-uniformity may be severe and will degrade the performance of classical algorithms. Therefore, it is important to solve the nonuniform noise problem for sparse array.

ML methods are classical algorithms to deal with the nonuniform noise problem in DOA estimation. The ML methods can avoid the eigen-decomposition, and the corresponding noise matrix can be estimated and eliminated to improve the accuracy of DOA estimation. A deterministic non-uniform ML estimator is developed in [12], and the Cramér-Rao bounds (CRB) of both the deterministic and stochastic signal model are given. However, the stochastic ML DOA estimator is not derived in the literature. On the basis of this paper, Chen et al. propose a stochastic ML estimator in non-uniform noise [13]. All of the ML methods need iterative optimization, which brings high computation complexity. Though Liao B. et al. improve the method to reduce the computational complexity $[14,15]$, stepwise concentration is still essential and time consuming. Besides, there is a chance that the obtained result is a local optimum but not a global one.

Subspace based algorithms are the dominant methods in DOA estimation. Many methods are proposed to solve the non-uniform noise problem by eliminating the main diagonal elements or averaging these main diagonal elements [15]. Based on the matrix completion theory, the estimation of the noise-free covariance matrix is solved by the rank minimization [16], and nuclear norm minimization is employed to transform the non-convex problem to a convex one. This method avoids the iterative optimization; thus it is computationally efficient. In [17], the covariance matrix is divided into some sub-matrices and the noise covariance is estimated based on the relationship between the divided submatrices. However the number of detectable targets is limited.

In recent years, the DOA estimation based on sparse recovery method has aroused a lot of attention $[18,19]$. To solve the non-uniform noise problem in sparse signal recovery, $\mathrm{He} \mathrm{Z}$. et al. propose a novel covariance sparsity aware (CSA) DOA estimation method in [20], which directly removes the unknown noise variances by a linear transformation. This method improves the accuracy, but it also causes the loss of effective aperture.

In summary, the current methods have not completely settled the non-uniform noise problem. Based on the above analysis, a novel diagonal reloading (DR) DOA estimation algorithm for nested array is proposed in unknown nonuniform noise. The elements in the main diagonal of the received data covariance matrix are eliminated, and the smallest $M N-K$ eigenvalues of the revised matrix are obtained and averaged to estimate the sum of the signal power. Then the estimated sum is filled into the main diagonal of the revised matrix for estimating the signal covariance matrix. Different from the method of completely removing the received data which contains noise, the proposed method only eliminates the negative effect of noise while keeping the useful information of the signal matrix.

The remainder of this paper is organized as follows. The nested MIMO radar structure and the corresponding signal model are introduced in Section 2. The proposed method is derived in Section 3. Several simulations are conducted in Section 4 to validate the performance of the proposed method and conclusions are drawn in Section 5.

\section{Signal Model for Nested MIMO Radar}

Suppose the transmitter and receiver of the MIMO radar are all parallel linear arrays with the sensor numbers $M$ and $N$, respectively. The code length and wavelength of each signal are $L(L<N)$ and $\lambda$, respectively. $\mathbf{S}=\left[\mathbf{s}_{1}, \mathbf{s}_{2}, \ldots\right.$, $\left.\mathbf{s}_{M}\right]^{T}$ is the transmitting signal matrix, among which $\mathbf{s}_{m}=$ $\left[\mathbf{s}_{m}(1), \mathbf{s}_{m}(2), \ldots, \mathbf{s}_{m}(L)\right]^{T}$ is the $m$ th transmitting signal. $[\cdot]^{T}$ denotes the transpose. The number of far field targets is $K$ and their DOAs are $\theta_{k}(k=1,2, \ldots, K)$. The received signal vector of a single snapshot is

$$
\mathbf{Y}=\sum_{k=1}^{K} \eta_{k} \mathbf{a}_{r}\left(\theta_{k}\right) \mathbf{a}_{t}^{T}\left(\theta_{k}\right) \mathbf{S}+\mathbf{U},
$$

where $\mathbf{Y}=\left[\mathbf{Y}_{1}, \mathbf{Y}_{2}, \ldots, \mathbf{Y}_{N}\right]^{T}$ and $\mathbf{Y}_{n}$ is the received signal of the $n$th sensor. $\mathbf{a}_{r}\left(\theta_{k}\right)=\left[1, e^{j f_{r 1}\left(\theta_{k}\right)}, \ldots, e^{j f_{r N}\left(\theta_{k}\right)}\right]$ and $\mathbf{a}_{t}\left(\theta_{k}\right)=$ $\left[1, e^{j f_{t 1}\left(\theta_{k}\right)}, \ldots, e^{j f_{t M}\left(\theta_{k}\right)}\right]$ are the transmitting and receiving steering vector respectively. $f_{r n}\left(\theta_{k}\right)$ and $f_{t m}\left(\theta_{k}\right)$ are functions with respect to $\theta_{k}$ for given coordinates of the sensors. $\mathbf{U}=$ $\left[\begin{array}{llll}\mathbf{u}_{1} & \mathbf{u}_{2} & \ldots & \mathbf{u}_{N}\end{array}\right] \in \mathbb{C}^{N \times L}$ denotes the noise matrix, and $\mathbf{u}_{n}=$ $\left[\mathbf{u}_{n}(1), \mathbf{u}_{n}(2), \ldots, \mathbf{u}_{n}(L)\right]$. The noises are assumed to be nonuniform and uncorrelated with each other, namely, $\mathbf{u}_{i} \neq \mathbf{u}_{j}$, $E\left[\mathbf{u}_{i} \mathbf{u}_{j}^{H}\right]=0(i, j=1,2, \ldots, N, i \neq j)$.

Conducting matched filtering with the $M$ orthogonal transmitting waveforms in each receiver, we obtain that

$$
\begin{aligned}
\mathbf{z}(t) & =\operatorname{vec}\left(\frac{1}{L} \mathbf{Y} \mathbf{S}^{H}\right) \\
& =\operatorname{vec}\left[\frac{1}{L}\left(\sum_{k=1}^{K} \eta_{k} \mathbf{a}_{r}\left(\theta_{k}\right) \mathbf{a}_{t}^{T}\left(\theta_{k}\right) \mathbf{S}+\mathbf{U}\right) \mathbf{S}^{H}\right],
\end{aligned}
$$

where $\operatorname{vec}(\cdot)$ is the operation to rearrange matrix into one column and $(\cdot)^{H}$ denotes Hermitian transpose. As the transmitting signals are orthogonal with each other, $\mathbf{z}(t)$ can be simplified as

$$
\begin{aligned}
\mathbf{z}(t) & =\sum_{k=1}^{K} \eta_{k}\left(\mathbf{a}_{r}\left(\theta_{k}\right) \otimes \mathbf{a}_{t}\left(\theta_{k}\right)\right)+\mathbf{v}(t) \\
& =\sum_{k=1}^{K} \eta_{k} \mathbf{a}_{\mathrm{tr}}\left(\theta_{k}\right)+\mathbf{v}(t)=\mathbf{A}_{\mathrm{tr}} \boldsymbol{\eta}(t)+\mathbf{v}(t)
\end{aligned}
$$

wherein $\mathbf{A}_{\mathrm{tr}}=\left[\mathbf{a}_{\mathrm{tr}}\left(\theta_{1}\right), \mathbf{a}_{\mathrm{tr}}\left(\theta_{2}\right), \ldots, \mathbf{a}_{\mathrm{tr}}\left(\theta_{K}\right)\right]$ is the steering matrix of the sum co-array, and $\otimes$ denotes the Kronecker product. $\boldsymbol{\eta}(t)=\left[\eta_{1}, \eta_{2}, \ldots, \eta_{K}\right]^{T}$ is the reflection coefficient of the $K$ targets. $\mathbf{v}=\operatorname{vec}\left(\mathbf{U S} S^{H} / L\right)$ is the output of noise after matched filtering. Define $\mathbf{v}_{n m}$ as the element in the $n$th row and $m$ th column of $N \times M$ matrix $\mathbf{U S}^{H} / L$, i.e.,

$$
\mathbf{v}_{n m}=\mathbf{u}_{n} \times \mathbf{s}_{m}^{*}=\sum_{l=1}^{L} \mathbf{u}_{n}(l) \times \mathbf{s}_{m}^{*}(l)
$$


As both $\mathbf{u}_{n}$ and $\mathbf{s}_{m}$ are unique vectors, namely, $\mathbf{u}_{1} \neq \mathbf{u}_{2} \cdots \neq$ $\mathbf{u}_{N}$ and $\mathbf{s}_{1} \neq \mathbf{s}_{2} \ldots \neq \mathbf{s}_{M}$, their product $\mathbf{v}_{n m}(n=1,2, \ldots$, $N, m=1,2, \ldots, M)$ is unique, too. Therefore, the noise remains non-uniform after matched filtering.

When the radar transmits $P$ pulses, we have

$$
\mathbf{Z}=[\mathbf{z}(1), \mathbf{z}(2), \ldots, \mathbf{z}(P)],
$$

where $\mathbf{z}(p),(p=1,2, \ldots, P)$ is the receiving signal corresponding to the $p$ th transmitting pulse, that is,

$$
\mathbf{z}(p)=\sum_{k=1}^{K} \eta_{k} \mathbf{a}_{\mathrm{tr}}\left(\theta_{k}\right)+\mathbf{v}(p)=\mathbf{A}_{\mathrm{tr}} \boldsymbol{\eta}(p)+\mathbf{v}(p)
$$

As a result, the total received signal can be expressed as

$$
\mathbf{Z}=\mathbf{A}_{\mathrm{tr}} \mathbf{B}+\mathbf{V}
$$

where $\mathbf{B}=[\boldsymbol{\eta}(1), \boldsymbol{\eta}(2), \ldots, \boldsymbol{\eta}(P)]$ is the $K \times P$ matrix of the reflection coefficient and $\mathbf{V}=[\mathbf{v}(1), \mathbf{v}(2), \ldots, \mathbf{v}(P)]$ is the $M N \times P$ matrix of the noise.

Since the noise is uncorrelated with each other, the covariance of the array is

$$
\mathbf{R}=E\left[\mathbf{Z Z}^{H}\right]=\mathbf{A}_{\mathrm{tr}} \mathbf{R}_{s} \mathbf{A}_{\mathrm{tr}}^{H}+\mathbf{Q},
$$

where $E[\cdot]$ denotes the mathematical expectation. $\mathbf{R}_{s}=$ $\operatorname{diag}\left(\sigma_{1}^{2}, \sigma_{2}^{2}, \ldots, \sigma_{K}^{2}\right)$, and $\sigma_{k}^{2}(k=1,2, \ldots, K)$ is the reflection coefficient covariance of the $k$ th target. $\mathbf{Q}=\operatorname{diag}\left[\rho_{1}^{2}, \rho_{2}^{2}, \ldots\right.$, $\left.\rho_{M N}^{2}\right] ; \operatorname{diag}\left[\rho_{1}^{2}, \rho_{2}^{2}, \ldots, \rho_{M N}^{2}\right]$ denotes a diagonal matrix with $\rho_{1}^{2}, \rho_{2}^{2}, \ldots, \rho_{M N}^{2}$ in its main diagonal. $\rho_{i}^{2}(i=1,2, \ldots, M N)$ is the covariance of the noise and they are unequal with each other. Vectorize $\mathbf{R}$ to expand the effective DOFs, i.e.,

$$
\begin{aligned}
\mathbf{y} & \triangleq \operatorname{vec}(\mathbf{R})=\operatorname{vec}\left[\sum_{k=1}^{K} \sigma_{k}^{2} \mathbf{a}_{\mathrm{tr}}\left(\theta_{k}\right) \mathbf{a}_{\mathrm{tr}}^{H}\left(\theta_{k}\right)\right]+\operatorname{vec}(\mathbf{Q}) \\
& =\left[\mathbf{A}_{\mathrm{tr}}^{*}(\theta) \odot \mathbf{A}_{\mathrm{tr}}(\theta)\right] \mathbf{b}+\mathbf{1}_{n},
\end{aligned}
$$

where $\odot$ denotes the Khatri-Rao product operation, and $\mathbf{b}=$ $\left[\sigma_{1}^{2}, \sigma_{2}^{2}, \ldots, \sigma_{K}^{2}\right]^{T} . \mathbf{1}_{n}=\left[\rho_{1}^{2} \mathbf{e}_{1}^{T}, \ldots, \rho_{M N}^{2} \mathbf{e}_{M N}^{T}\right]^{T}$, where $\mathbf{e}_{i}(i=$ $1, \ldots, M N)$ is $M N \times 1$ column vector of all zeroes except a 1 in the $i$ th entry. The obtained difference co-array is

$$
\begin{aligned}
& \mathbf{A}_{\mathrm{tr}}^{*}(\theta) \odot \mathbf{A}_{\mathrm{tr}}(\theta) \\
& \quad \triangleq\left[\mathbf{a}_{\mathrm{tr}}^{*}\left(\theta_{1}\right) \otimes \mathbf{a}_{\mathrm{tr}}\left(\theta_{1}\right), \ldots, \mathbf{a}_{\mathrm{tr}}^{*}\left(\theta_{K}\right) \otimes \mathbf{a}_{\mathrm{tr}}\left(\theta_{K}\right)\right]
\end{aligned}
$$

Let $\widetilde{\mathbf{A}}(\theta)=\mathbf{A}_{\mathrm{tr}}^{*}(\theta) \odot \mathbf{A}_{\mathrm{tr}}(\theta)$. Compared with $\mathbf{A}(\theta), \widetilde{\mathbf{A}}(\theta)$ expands the effective DOFs by constructing the difference coarray, whose virtual sensors' positions are expressed as

$$
\left\{\left(x_{t, m 1}+x_{r, n 1}\right)-\left(x_{t, m 2}+x_{r, n 2}\right)\right\}
$$

where $1 \leq m_{1}, m_{2} \leq M, 1 \leq n_{1}, n_{2} \leq N, x_{t, m}$ and $x_{r, n}$ are the sensors' positions of transmitting array and receiving array, respectively. Now the question turns to maximize the number of unique difference results in (11). Here we adopt a new structure of nested MIMO array to settle the problem [11].
First, the geometry of the receiver is designed as a two level nested array, whose locations are

$$
\left\{u_{t, m}\right\}=\left\{a_{m} \mid m=1,2, \ldots, M\right\},
$$

where $\left\{a_{m}\right\}_{m=1}^{M}$ is the location of the nested array. This is a hole free array after difference. The number of DOFs in the difference co-array of the transmitting array is $f_{M}$, which is summarized as

$$
f_{M}= \begin{cases}\frac{M^{2}}{2}+M-1 & \text { if } M \text { is even } \\ \frac{(M+1)^{2}}{2}-1 & \text { if } M \text { is odd }\end{cases}
$$

The receiving array is constructed as another nested array with a large unit inter-element spacing $J$, whose locations are denoted by

$$
\left\{u_{r, n}\right\}=\left\{b_{n} \cdot J \mid n=1,2, \ldots, N\right\}
$$

$\left\{b_{n}\right\}_{n=1}^{N}$ is the location sequence of the nested array.

In all, the sum co-array of the MIMO radar can be expressed as

$$
\begin{aligned}
& \left\{u_{t, m}+u_{r, n}\right\} \\
& \quad=\left\{a_{m}+b_{n} \cdot J \mid m=1,2, \ldots, M, n=1,2, \ldots, N\right\}
\end{aligned}
$$

The location of the difference co-array is

$$
\begin{aligned}
& \left\{a_{m}+b_{n} \cdot J-a_{m^{\prime}}-b_{n^{\prime}} \cdot J\right\} \\
& \quad=\left\{\left(a_{m}-a_{m^{\prime}}\right)+\left(b_{n}-b_{n^{\prime}}\right) \cdot J\right\},
\end{aligned}
$$

where $m, m^{\prime}=1,2, \ldots, M, n, n^{\prime}=1,2, \ldots, N$. If $J=f_{M}$, a hole free ULA is obtained with expanded DOFs. If the number of sensors satisfies

$$
F=M+N
$$

then the best values of $M$ and $N$ are

$$
\begin{aligned}
& M=N=\frac{F}{2}, \quad \text { if } F \text { is even } \\
& M=\frac{(F-1)}{2}, \\
& N=\frac{(F+1)}{2}
\end{aligned}
$$

if $F$ is odd

Correspondingly, we obtain the optimal geometry of the transmitting and receiving nested array. For example, if $F$ is even, $M=N=F / 2$, the final obtained DOF is

$$
\begin{aligned}
f & =f_{M} \cdot f_{N}=\left(\frac{M^{2}}{2}+M-1\right) \cdot\left(\frac{N^{2}}{2}+N-1\right) \\
& =\left(\frac{F^{2}}{8}+\frac{F}{2}-1\right)^{2}
\end{aligned}
$$




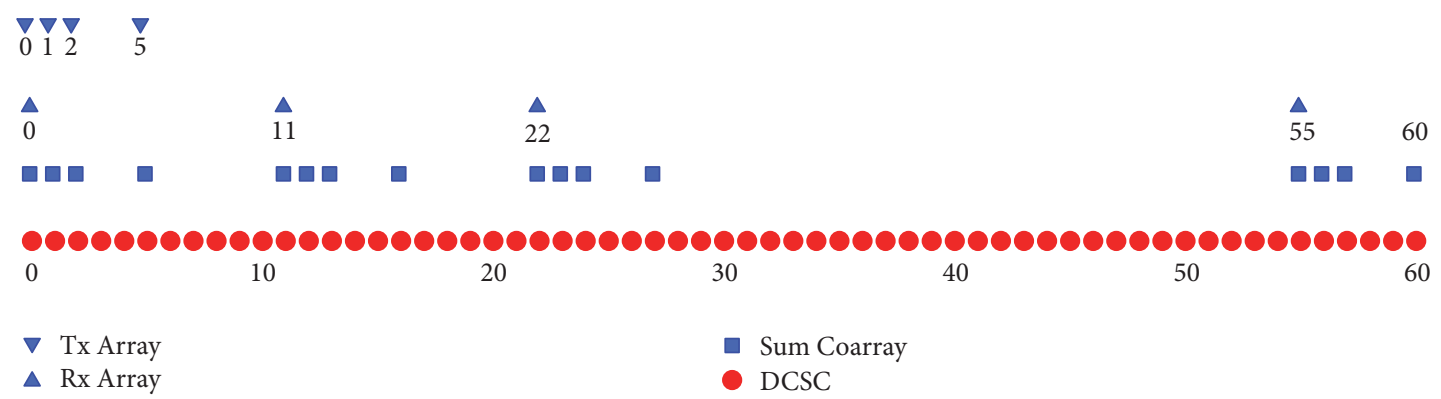

FIGURE 1: Structure of the nested MIMO radar array with a four-element transmitting (Tx) array and a four-element receiving (Rx) array $(J=11)$.

We can find that, by this kind of structure, the nested array can provide $\mathrm{O}\left(F^{4}\right)$ DOFs with only $F$ sensors. Figure 1 shows the structure of the nested MIMO radar array with a fourelement transmitting (Tx) array and a four-element receiving $(\mathrm{Rx})$ array. The numbers under the sensors indicate their positions. It should be mentioned that only the nonnegative part of the DCSC is given in this figure. Compared with the former structure, the number of DOFs is greatly expanded using the same number of sensors, and the DOA estimation accuracy will be highly promoted.

The application of nested MIMO array effectively improves the virtual aperture; however, it also brings another problem. Due to the large spacing between the receiving array, the received noise of different sensors no more satisfy the uniform assumption. Therefore $\mathbf{Q}$ in (8) will turn to a non-uniform diagonal matrix and the DOA estimation performance will degrade significantly. In this paper, we will analyze the negative effect of non-uniform noise and propose a novel diagonal recovery algorithm to solve this problem.

\section{Diagonal Reloading Based DOA Estimation Algorithm}

3.1. Negative Effect Analysis of Non-Uniform Noise. In ideal situation, there is no noise. So the matrix $\mathbf{Q}$ in (8) shall be equal to $\mathbf{0}$. As a result, $\mathbf{R}=\mathbf{A}_{\mathrm{tr}} \mathbf{R}_{\mathrm{s}} \mathbf{A}_{\mathrm{tr}}^{H}$. Conducting eigendecomposition with $\mathbf{A}_{\mathrm{tr}} \mathbf{R}_{\mathrm{s}} \mathbf{A}_{\mathrm{tr}}^{H}$, we have

$$
\mathbf{A}_{\mathrm{tr}} \mathbf{R}_{\mathrm{s}} \mathbf{A}_{\mathrm{tr}}^{H}=\mathbf{U}_{1} \boldsymbol{\Sigma}_{1} \mathbf{U}_{1}^{H}+\mathbf{U}_{2} \boldsymbol{\Sigma}_{2} \mathbf{U}_{2}^{H},
$$

where $\mathbf{U}_{1}$ consists of eigenvectors corresponding to the $K$ largest eigenvalues and $\mathbf{U}_{2}$ is consisted of the rest of $M N-K$ eigenvectors corresponding to the other $M N-K$ smaller eigenvalues. However, noise is inevitable and will degrade the DOA performance. It can be explained from the structure of the covariance. Let $\mathbf{a}_{h} \triangleq\left[a_{h}\left(\theta_{1}\right), a_{h}\left(\theta_{2}\right), \ldots, a_{h}\left(\theta_{K}\right)\right]$ represent the $h$ th row of $\mathbf{A}_{\mathrm{tr}} \cdot a_{h}\left(\theta_{k}\right)=e^{j f_{h}(\theta)}$, wherein $f_{h}\left(\theta_{k}\right)$ is function with respect to $\theta_{k}$ of the virtual sensors. Therefore $\left|a_{h}\left(\theta_{k}\right)\right|^{2}=$ 1 , and the elements in the main diagonal of $\mathbf{A}_{\mathrm{tr}} \mathbf{R}_{s} \mathbf{A}_{\mathrm{tr}}^{H}$ can be expressed as

$$
\left[\mathbf{A}_{\mathrm{tr}} \mathbf{R}_{s} \mathbf{A}_{\mathrm{tr}}^{H}\right]_{h, h}=\mathbf{a}_{h} \mathbf{R}_{s} \mathbf{a}_{h}^{H}=\sum_{k=1}^{K} \sigma_{k}^{2}\left|a_{h}\left(\theta_{k}\right)\right|^{2}=\sum_{k=1}^{K} \sigma_{k}^{2}
$$

It can be found that $\left[\mathbf{A}_{\mathrm{tr}} \mathbf{R}_{s} \mathbf{A}_{\mathrm{tr}}^{H}\right]_{h, h}$ equals the sum value of all targets' power. As $\mathbf{Q}$ is a diagonal matrix, it will be added to the main diagonal of $\mathbf{A}_{\mathrm{tr}} \mathbf{R}_{s} \mathbf{A}_{\mathrm{tr}}^{H}$ and only affect the element $\left[\mathbf{A}_{\mathrm{tr}} \mathbf{R}_{s} \mathbf{A}_{\mathrm{tr}}^{H}\right]_{h, h}$.

If the noise is uniform, namely, $\rho_{1}^{2}=\rho_{2}^{2}=\cdots=\rho_{M N}^{2}=\rho^{2}$ and $\mathbf{Q}=\rho^{2} \mathbf{I}_{M N}$, we have

$$
\begin{aligned}
\mathbf{R} & =\mathbf{A}_{\mathrm{tr}} \mathbf{R}_{s} \mathbf{A}_{\mathrm{tr}}^{H}+\mathbf{Q}=\mathbf{U}\left(\boldsymbol{\Sigma}+\rho^{2} \mathbf{I}_{M N}\right) \mathbf{U}^{H} \\
& =\mathbf{U}_{1}\left(\boldsymbol{\Sigma}_{1}+\rho^{2} \mathbf{I}_{K}\right) \mathbf{U}_{1}^{H}+\mathbf{U}_{2}\left(\rho^{2} \mathbf{I}_{M N-K}\right) \mathbf{U}_{2}^{H}
\end{aligned}
$$

It can be found that the elements in the main diagonal remain uniform, so it can be seen as the change of signal power and the structure of the main diagonal has not been changed. The matrix $\mathbf{U}_{1}$ corresponding to the eigenvectors associated with the $K$ largest eigenvalues of $\mathbf{R}$ remains the same as before. It is the same with the noise subspace $\mathbf{U}_{2}$. However, when the noise is non-uniform, the elements in the main diagonal no longer equal to each other. As $\mathbf{Q}=\rho^{2} \mathbf{I}_{M N}$ no longer holds, $\mathbf{A}_{\mathrm{tr}} \mathbf{R}_{s} \mathbf{A}_{\mathrm{tr}}^{H}+\mathbf{Q}$ no longer equals to $\mathbf{U}(\boldsymbol{\Sigma}+$ $\left.\rho^{2} \mathbf{I}_{M N}\right) \mathbf{U}^{H}$. Correspondingly, the signal subspace $\mathbf{U}_{1}$ and the noise subspace $\mathbf{U}_{2}$ will be changed and the performance of DOA estimation will degrade significantly.

3.2. Diagonal Reloading (DR) Algorithm. In [15], the diagonal elements of the covariance matrix $\mathbf{R}$ are completely eliminated (as shown in (23)). As the covariance of noise is a diagonal matrix, it will be removed from $\mathbf{R}$. However, the main diagonal elements of the signal covariance will also be eliminated:

$$
\begin{aligned}
\breve{\mathbf{R}} & =\mathbf{R}-D(\mathbf{R})=\mathbf{A}_{\mathrm{tr}} \mathbf{R}_{s} \mathbf{A}_{\mathrm{tr}}^{H}+\mathbf{Q}-D\left(\mathbf{A}_{\mathrm{tr}} \mathbf{R}_{\mathrm{s}} \mathbf{A}_{\mathrm{tr}}^{H}+\mathbf{Q}\right) \\
& =\mathbf{A}_{\mathrm{tr}} \mathbf{R}_{s} \mathbf{A}_{\mathrm{tr}}^{H}-D\left(\mathbf{A}_{\mathrm{tr}} \mathbf{R}_{s} \mathbf{A}_{\mathrm{tr}}^{H}\right),
\end{aligned}
$$

where $D\left(\mathbf{A}_{\mathrm{tr}} \mathbf{R}_{\mathrm{s}} \mathbf{A}_{\mathrm{tr}}^{H}\right)$ denotes the operation to construct a diagonal matrix whose main diagonal elements are the same as $\mathbf{A}_{\mathrm{tr}} \mathbf{R}_{\mathrm{s}} \mathbf{A}_{\mathrm{tr}}^{H}$. The main diagonal contains the power of target signal. If they are replaced with zeroes as (23), it will certainly lose valuable information. An improved method which only eliminates noise and keeps the elements of signal is thus proposed.

$$
\begin{aligned}
& \text { Set } \gamma=\sum_{k=1}^{K} \sigma_{k}^{2} \text {; there is } \\
& \qquad D\left(\mathbf{A}_{\mathrm{tr}} \mathbf{R}_{s} \mathbf{A}_{\mathrm{tr}}^{\mathrm{H}}\right)=\gamma \mathbf{I}_{M}
\end{aligned}
$$


Therefore, $\breve{\mathbf{R}}$ can be expressed as

$$
\begin{aligned}
\breve{\mathbf{R}} & =\mathbf{A}_{\mathrm{tr}} \mathbf{R}_{s} \mathbf{A}_{\mathrm{tr}}^{H}-\gamma \mathbf{I}_{M}=\mathbf{U}\left(\boldsymbol{\Sigma}-\gamma \mathbf{I}_{M}\right) \mathbf{U}^{H} \\
& =\mathbf{U}_{1}\left(\boldsymbol{\Sigma}_{1}-\gamma \mathbf{I}_{K}\right) \mathbf{U}_{1}^{H}+\mathbf{U}_{2}\left(-\gamma \mathbf{I}_{M N-K}\right) \mathbf{U}_{2}^{H}
\end{aligned}
$$

We can find that the smallest $M N-K$ eigenvalues corresponding to $\mathbf{U}_{2}$ happen to be $-\gamma$, which means that $\gamma$ can be estimated by the average of $M N-K$ smallest eigenvalues. Based on this principle, we first conduct eigen-decomposition of $\breve{\mathbf{R}}$ as (25) and get an estimation of $\gamma$, then the elements in main diagonal of $\breve{\mathbf{R}}$ can be replaced with $\widehat{\gamma} \mathbf{I}_{M}$, namely,

$$
\widetilde{\mathbf{R}}=\breve{\mathbf{R}}+\widehat{\gamma} \mathbf{I}_{M}
$$

where $\widehat{\gamma}$ is the estimation of $\gamma \cdot \widetilde{\mathbf{R}}$ is an estimate of $\mathbf{A}_{\mathrm{tr}} \mathbf{R}_{s} \mathbf{A}_{\mathrm{tr}}^{H}$. If the estimation of $\gamma$ is accurate, namely, $\widehat{\gamma}=\gamma$, then $\widetilde{\mathbf{R}}=$ $\mathbf{A}_{\mathrm{tr}} \mathbf{R}_{s} \mathbf{A}_{\mathrm{tr}}^{H}$, and the covariance of the signal matrix can be accurately reconstructed.

In conclusion, the proposed method removes the negative influence of noise while keeping the main diagonal elements of signal matrix.

3.3. Sparse Signal Recovery. It can be seen that the effective DOFs have obvious improvement after vectorization, leading to a more precise estimation. Now we can divide the whole space to grids with a series of angles $\widetilde{\theta}_{1}, \widetilde{\theta}_{2}, \ldots, \widetilde{\theta}_{N_{s}}$. The grid angles are sufficiently dense and each of them represents a possible target direction. Since the actual targets only appear in a few angles and the coming signals are sparse in the whole space, the nonzero elements among the whole grid points correspond to the real DOAs. Set $\widetilde{\mathbf{A}}(\widetilde{\theta})=$ $\left[\mathbf{a}\left(\widetilde{\theta}_{1}\right), \mathbf{a}\left(\widetilde{\theta}_{2}\right), \ldots, \mathbf{a}\left(\widetilde{\theta}_{N_{s}}\right)\right] \in \mathbb{C}^{f \times N_{s}}$ as the over-complete basis, where $f$ is given by (19). The covariance can be expressed in a sparse way as

$$
\widetilde{\mathbf{y}}=\widetilde{\mathbf{A}}(\widetilde{\theta}) \mathbf{b}_{s}+\mathbf{1}_{n}
$$

where $\mathbf{b}_{s}=\left[\begin{array}{llll}b_{1} & b_{2} & \ldots & b_{N_{s}}\end{array}\right]^{T}$ is the sparse vector with rank $K$.

$$
\begin{aligned}
& b_{i}= \begin{cases}b_{k}, & \tilde{\theta}_{i} \in\left\{\theta_{1}, \theta_{2}, \ldots, \theta_{K}\right\} \\
0, & \tilde{\theta}_{i} \notin\left\{\theta_{1}, \theta_{2}, \ldots, \theta_{K}\right\},\end{cases} \\
& i=1,2, \ldots, N_{s}, k=1,2, \ldots, K
\end{aligned}
$$

Now the problem turns to recover the $K$ nonzero elements from $\widetilde{\mathbf{r}}_{s}$. Theoretically, we should use $l_{0}$ norm to solve the sparse recovery problem; however it is a non-convex, NPhard problem. The $l_{1}$ norm is usually used to relax this problem, namely,

$$
\begin{array}{ll}
\min _{\mathbf{b}_{s}} & \left\|\mathbf{b}_{s}\right\|_{1} \\
\text { s.t. } & \widetilde{\mathbf{y}}=\widetilde{\mathbf{A}}(\widetilde{\theta}) \mathbf{b}_{s}+\mathbf{1}_{n}
\end{array}
$$

Due to the existence of noise, the optimization problem should be expressed as

$$
\begin{array}{ll}
\min _{\boldsymbol{\eta}, \mathbf{b}_{s}} & \xi \mathbf{1}^{T} \boldsymbol{\eta}+\left\|\widetilde{\mathbf{y}}-\widetilde{\mathbf{A}}(\widetilde{\theta}) \mathbf{b}_{s}\right\|_{2}^{2} \leq \beta \\
\text { s.t. } & \boldsymbol{\eta} \geq \mathbf{0},
\end{array}
$$

where $\xi$ is the penalty factor and $\boldsymbol{\eta}$ is the sparse set we tend to solve.

In summary, the steps of the proposed methods are as follows:

(1) Construct covariance matrix of the received signal and remove the elements in the main diagonal as (23).

(2) Conduct the eigen-decomposition of $\breve{\mathbf{R}}$ and use the smallest $M N-K$ estimated eigenvalues to construct matrix $\widehat{\gamma} \mathbf{I}_{M}$.

(3) Fill the main diagonal of $\breve{\mathbf{R}}$ with $\widehat{\gamma}$ as (26).

(4) Vectorize the revised covariance matrix.

(5) Conduct sparse recovery of the target signal as (30).

\section{Simulation}

The first simulation is to validate the ability of MIMO and nested and MIMO nested array in distinguishing underestimated targets. The transmitter array and receiver array both have 4 sensors. We suppose that the targets are of the same power $\sigma^{2}$. The noise is modeled as white Gaussian process of power $\rho_{i}^{2}(i=1,2, \ldots, M N)$. To express the non-uniformity of noise, the worst noise power ratio (WNPR) is defined as $\mathrm{WNPR}=\rho_{\max }^{2} / \rho_{\min }^{2}$. As (3) shows, after matched filtering, the array has $M \times N=16$ distinct virtual receivers; the corresponding noise power of each virtual receiver is [30, 9 , $3,6,5,9,7,5,1,8,5,6,8,9,7,2]$. So in this simulation, the $\mathrm{WNPR}=30$. The average SNR is defined as

$$
\mathrm{SNR}=10 \log _{10}\left[\frac{1}{M N} \sum_{i=1}^{M N} \frac{\sigma^{2}}{\rho_{i}^{2}}\right] \mathrm{dB}
$$

Here we set the SNR as 0dB. Ten narrowband Gaussian signals coming from directions $\left[10^{\circ}, 15^{\circ}, 20^{\circ}, 25^{\circ}, 30^{\circ}, 35^{\circ}\right.$, $40^{\circ}, 45^{\circ}, 50^{\circ}, 55^{\circ}$ ] are received by the nested MIMO array. And the candidate direction grid is set as $0.1^{\circ}$. The number of snapshots is 200 .

Based on $l_{1}$ norm sparse recovery, the DOA estimation performance of different structures is compared in Figure 2. The MIMO array and the nested array cannot distinguish all the ten targets, but for nested MIMO array, all of the targets can be estimated accurately.

Next, several simulations are conducted under different conditions to examine the effectiveness of the proposed DR method. All of the results are obtained using 500 independent trials.

Simulation 2 compares the DOA estimation performance of different methods versus SNR. The number of targets and their directions are as simulation 1. The WNPR equals 30 and 


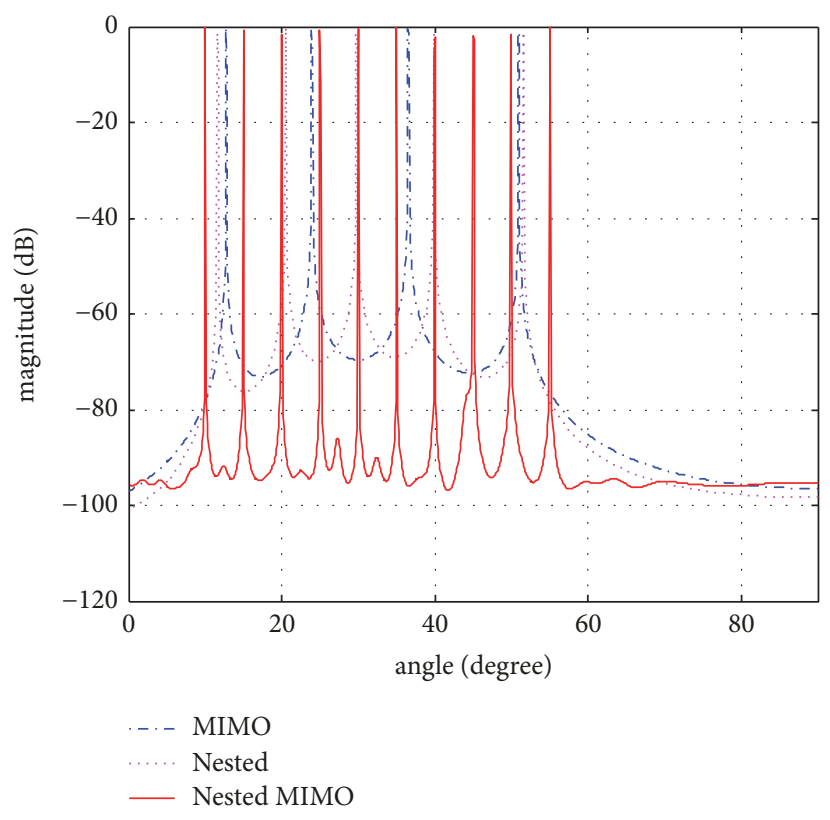

FIGURE 2: Spatial spectrums of different arrays.

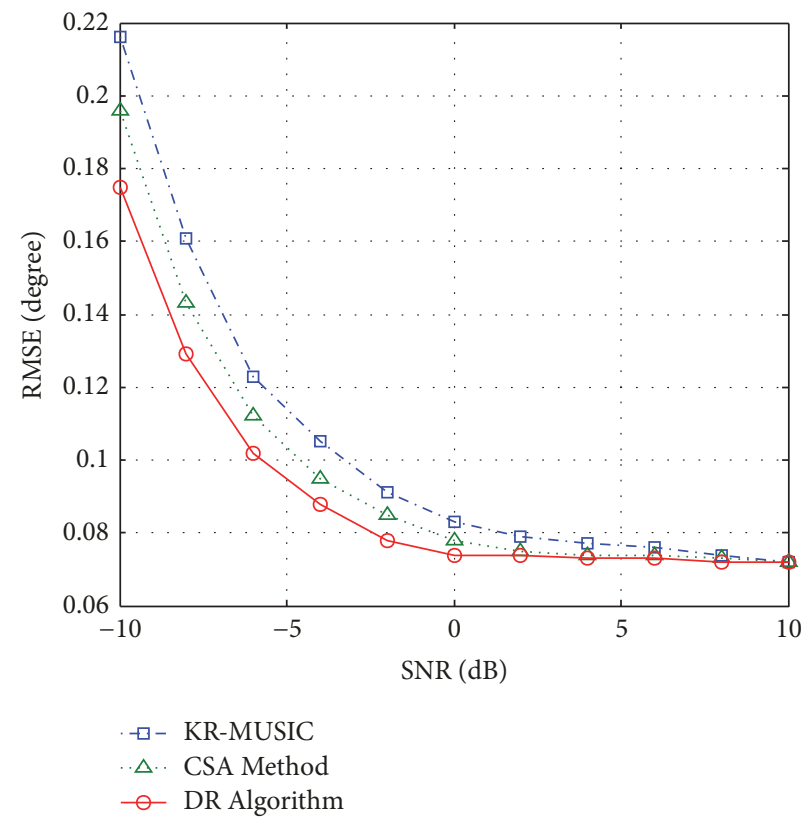

FIGURE 3: DOA estimation RMSEs versus SNR.

the number of snapshots is $N=200$. The root mean square error (RMSE) is defined as

$$
\mathrm{RMSE}=\left[\frac{1}{500 K} \sum_{k=1}^{K} \sum_{r=1}^{500}\left(\widehat{\theta}_{k}^{r}-\theta_{k}\right)^{2}\right]^{1 / 2}
$$

Figure 3 shows all the RMSEs of DOA estimation decrease with the increase of SNR. It can be found that the KR-MUSIC performs the worst, while the proposed method has obvious improvement in accuracy, especially in low SNR region. It is because that the power of noise is equivalent or even stronger than that of signal in low SNR condition. The nonuniformity of noise will obviously affect the results of eigendecomposition.

Simulation 3 compares the DOA estimation performance of different methods versus the number of snapshots. The number of targets and their directions are same as simulation 1 . $\mathrm{SNR}=-5 \mathrm{~dB}$ and the $\mathrm{WNPR}$ equals 30 . Figure 4 shows the RMSEs versus the number of snapshots. With the increase of the number of snapshots, the accuracy of all these methods gets improved. Besides the proposed method outperforms the KR-MUSIC and CSA method. 


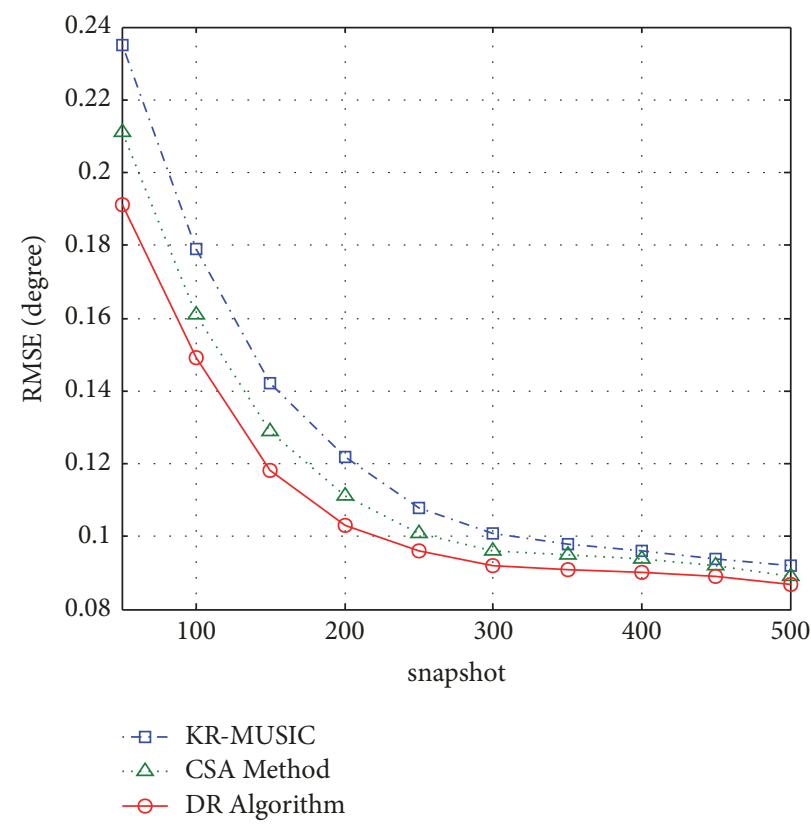

FIGURE 4: DOA estimation RMSEs versus snapshots.

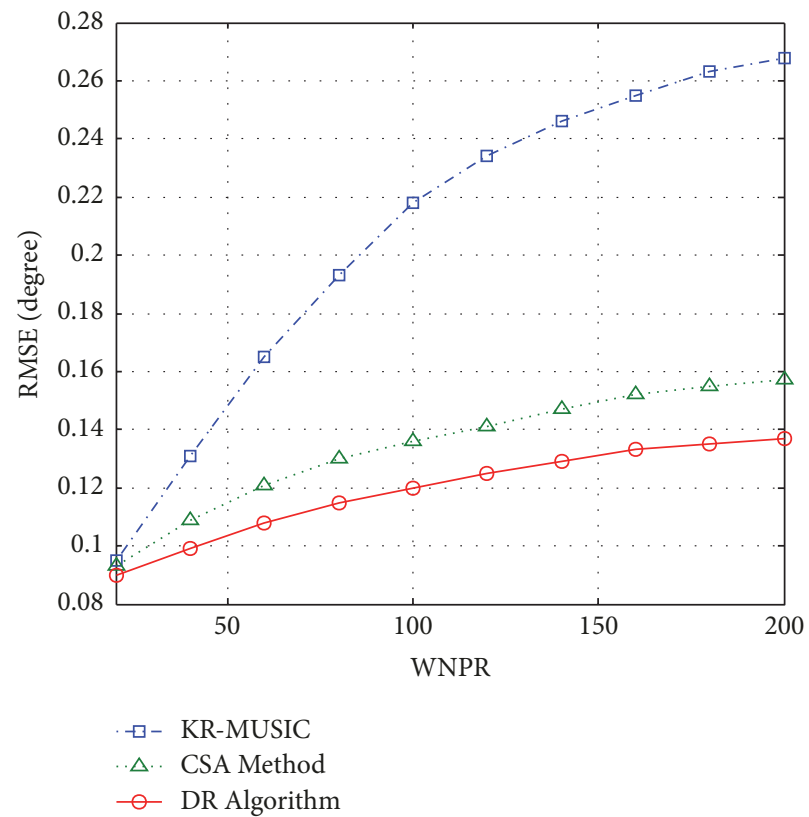

FIGURE 5: RMSEs of different DOA estimation methods versus WNPR.

Simulation 4 examines the DOA estimation performance of different methods under different levels of non-uniform noise. We assume that if the maximum DOA estimation error does not exceed $1^{\circ}$, the estimation is successful, and otherwise it fails. It can be seen from Figures 5 and 6 that, with the increase of WNPR, the RMSEs of these methods increase and the success probabilities decrease. In addition, compared with KR-MUSIC and the covariance sparsity aware (CSA) method in [20], the proposed method has better performance against the increase of WNPR.

\section{Conclusion}

In this paper, we proposed a novel DR based DOA estimation algorithm to solve the non-uniform noise problem for sparse array. First the elements in the main diagonal of received data covariance matrix were eliminated; then the smallest $M N-K$ eigenvalues of the revised matrix were obtained and averaged to estimate the sum of the signal power. Next the estimated sum was filled to the main diagonal of the revised matrix. In this way, the covariance of signal matrix was 


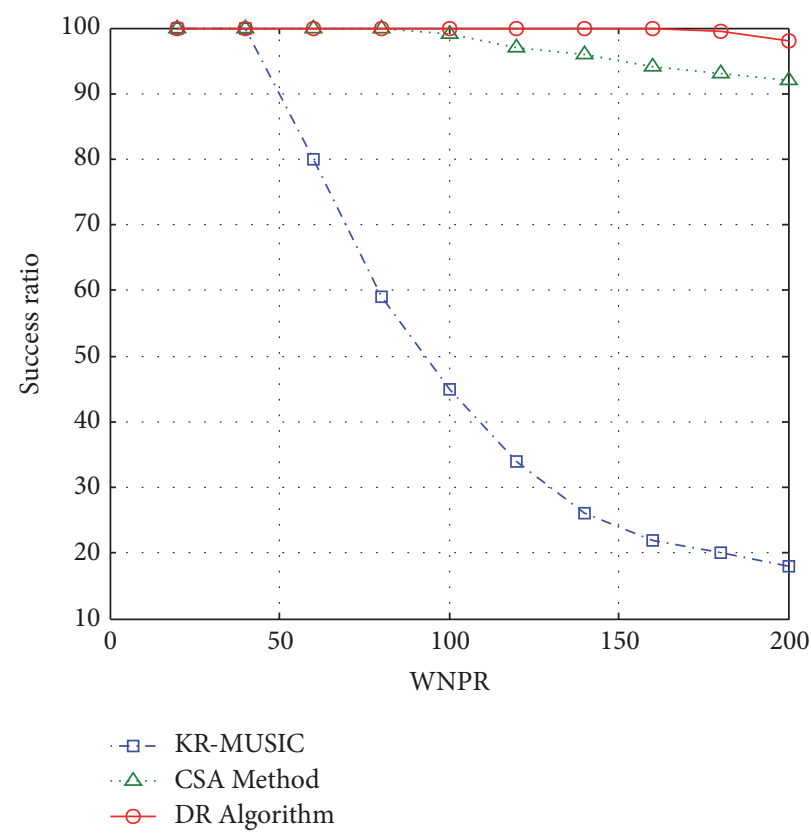

FIGURE 6: Success ratio of different DOA estimation methods versus WNPR.

recovered, where the negative effect of noise was eliminated without losing the useful information in the main diagonal of signal covariance matrix. Therefore the performance of proposed method was improved. Simulation results revealed that the proposed method not only made fully use of the DOF expansion of nested MIMO radar, but also suppressed the its non-uniform noise effect. Compared with the KR-MSUIC and the CSA method, the proposed method had better accuracy and higher success ratio. Furthermore, it could also be applied to arrays with closely spaced sensors.

\section{Data Availability}

The data used to support the findings of this study are included within the article.

\section{Conflicts of Interest}

The authors declare that there are no conflicts of interest regarding the publication of this paper.

\section{Acknowledgments}

This work was supported by the National Natural Science Foundation of China under Grant 61601504.

\section{References}

[1] Y. Dong and X. Chang, "Computationally Efficient 2D DOA Estimation for L-Shaped Array with Unknown Mutual Coupling," Mathematical Problems in Engineering, vol. 2018, pp. 1-9, 2018.

[2] Q. Shen, W. Liu, W. Cui, and S. Wu, "Underdetermined DOA estimation under the compressive sensing framework: A review," IEEE Access, vol. 4, pp. 8865-8878, 2016.
[3] R. O. Schmidt, "Multiple emitter location and signal parameter estimation," IEEE Transactions on Antennas and Propagation, vol. 34, no. 3, pp. 276-280, 1986.

[4] D. A. Linebarger, I. H. Sudborough, and I. G. Tollis, "Difference Bases and Sparse Sensor Arrays," IEEE Transactions on Information Theory, vol. 39, no. 2, pp. 716-721, 1993.

[5] C. S. Ruf, "Numerical Annealing of Low-Redundancy Linear Arrays," IEEE Transactions on Antennas and Propagation, vol. 41, no. 1, pp. 85-90, 1993.

[6] D. W. Bliss and K. W. Forsythe, "Multiple-input multipleoutput (MIMO) radar and imaging: degrees of freedom and resolution," in Proceedings of the Conference Record of the 37th IEEE Asilomar Conference on Signals, Systems and Computers (ACSSC '03), vol. 1, pp. 54-59, Pacific Grove, Calif, USA, November 2003.

[7] C. Zhu, H. Chen, and H. Shao, "Joint phased-MIMO and nested-array beamforming for increased degrees-of-freedom," International Journal of Antennas and Propagation, vol. 2015, Article ID 989517, 2015.

[8] J. Shi, G. Hu, X. Zhang et al., "Generalized Co-Prime MIMO Radar for DOA Estimation With Enhanced Degrees of Freedom," IEEE Sensors Journal, vol. 18, no. 3, pp. 1203-1212, 2018.

[9] D. C. C. Weng and P. P. Vaidyanathan, "Non-uniform sparse array design for active sensing," in Proceedings of the 2011 Conference Record of the Forty Fifth Asilomar Conference on Signals, Systems and Computers (ASILOMAR), pp. 1062-1066, IEEE, Pacific Grove, CA, USA, 2011.

[10] J. Shi, G. Hu, X. Zhang, F. Sun, and H. Zhou, "Sparsity-based two-dimensional DOA estimation for coprime array: from sum-difference coarray viewpoint," IEEE Transactions on Signal Processing, vol. 65, no. 21, pp. 5591-5604, 2017.

[11] M. Yang, L. Sun, X. Yuan, and B. Chen, "A New Nested MIMO Array with Increased Degrees of Freedom and Hole-free Difference Coarray," IEEE Signal Processing Letters, 2017.

[12] M. Pesavento and A. B. Gershman, "Maximum-likelihood direction-of-arrival estimation in the presence of unknown 
nonuniform noise," IEEE Transactions on Signal Processing, vol. 49, no. 7, pp. 1310-1324, 2001.

[13] C. Chen, F. Lorenzelli, R. E. Hudson et al., "Stochastic maximum-likelihood DOA estimation in the presence of unknown non-uniform noise," in Proceedings of the IEEE International Conference on Acoustics, vol. 56, pp. 2481-2484, 2008.

[14] B. Liao, S.-C. Chan, L. Huang, and C. Guo, "Iterative methods for subspace and DOA estimation in nonuniform noise," IEEE Transactions on Signal Processing, vol. 64, no. 12, pp. 3008-3020, 2016.

[15] B. Liao, L. Huang, C. Guo, and H. C. So, "New Approaches to Direction-of-Arrival Estimation with Sensor Arrays in Unknown Nonuniform Noise," IEEE Sensors Journal, vol. 16, no. 24, pp. 8982-8989, 2016.

[16] B. Liao, C. Guo, and H. C. So, "Direction-of-arrival estimation in nonuniform noise via low-rank matrix decomposition," in Proceedings of the 2017 22nd International Conference on Digital Signal Processing (DSP), pp. 1-4, London, UK, August 2017.

[17] Y. Wu, C. Hou, G. Liao, and Q. Guo, "Direction-of-arrival estimation in the presence of unknown nonuniform noise fields," IEEE Journal of Oceanic Engineering, vol. 31, no. 2, pp. 504-510, 2006.

[18] W. Si, X. Qu, Y. Jiang, and T. Chen, "Multiple sparse measurement gradient reconstruction algorithm for DOA estimation in compressed sensing," Mathematical Problems in Engineering, vol. 2015, Article ID 152570, 6 pages, 2015.

[19] Z. Q. He, Q. H. Liu, L. N. Jin, and S. Ouyang, "Low complexity method for DOA estimation using array covariance matrix sparse representation," IEEE Electronics Letters, vol. 49, no. 3, pp. 228-230, 2013.

[20] Z.-Q. He, Z.-P. Shi, and L. Huang, "Covariance sparsity-aware DOA estimation for nonuniform noise," Digital Signal Processing, vol. 28, no. 1, pp. 75-81, 2014. 


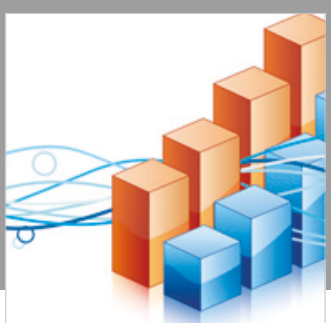

Advances in

Operations Research

\section{-n-m}
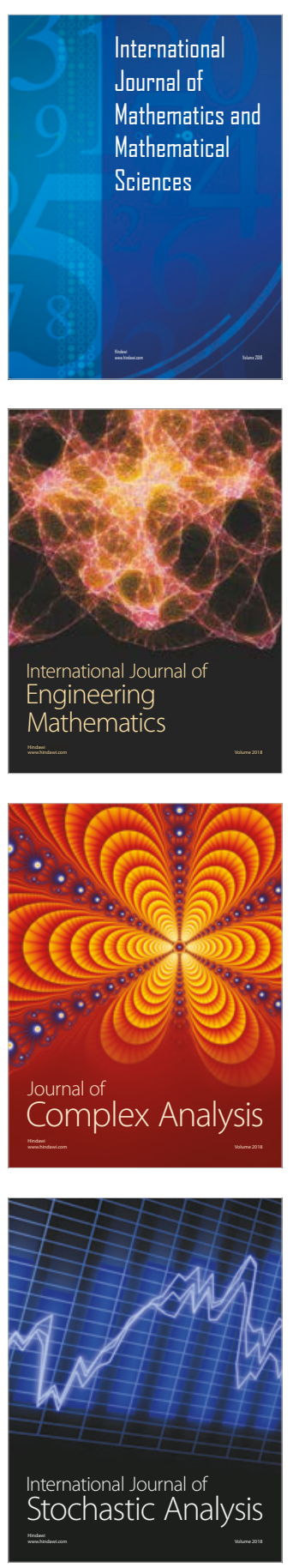
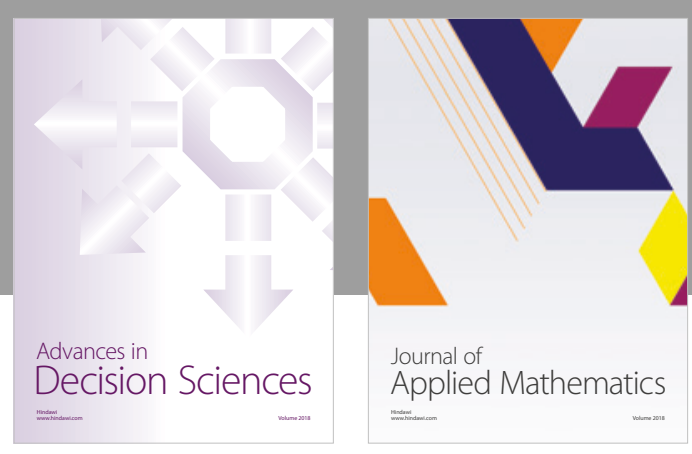

Journal of

Applied Mathematics
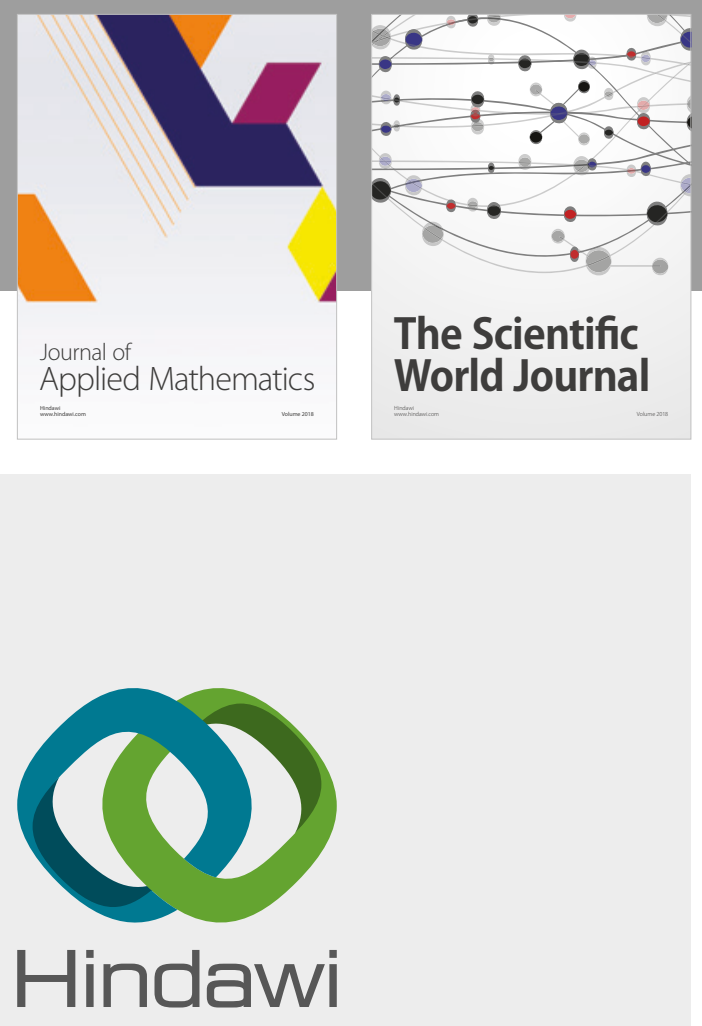

Submit your manuscripts at

www.hindawi.com

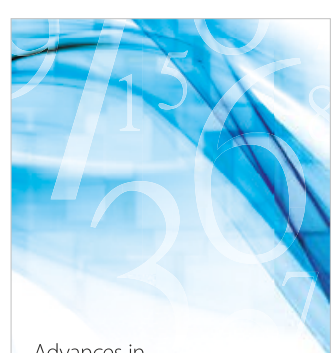

Advances in
Numerical Analysis
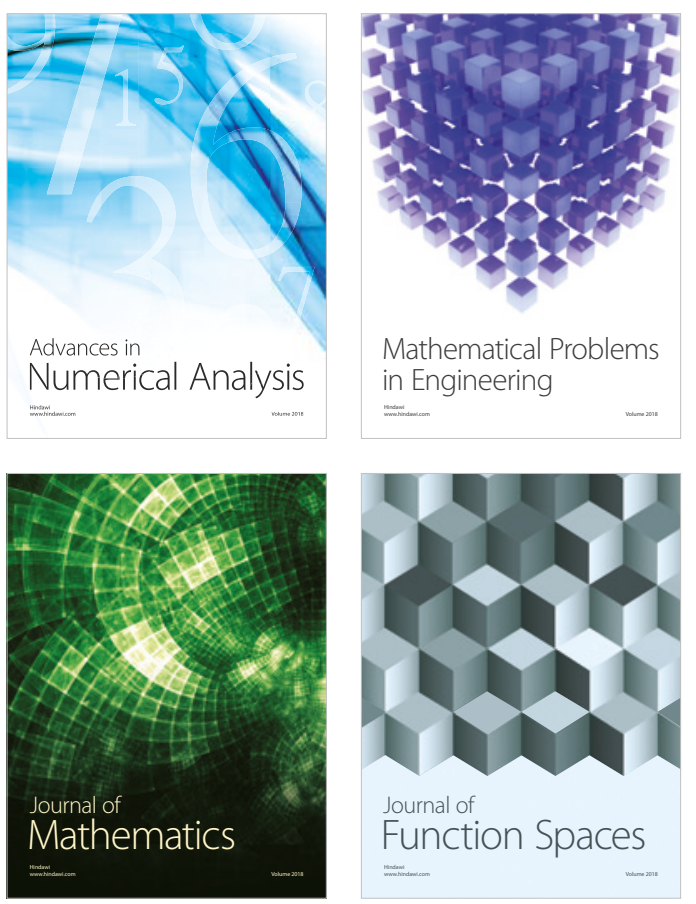

Mathematical Problems in Engineering

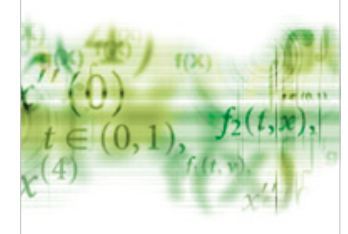

International Journal of

Differential Equations

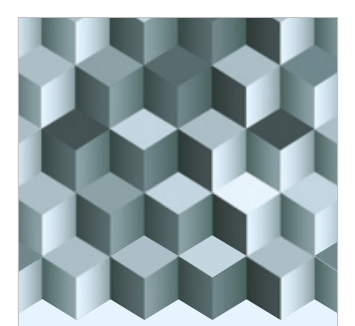

Journal of

Function Spaces

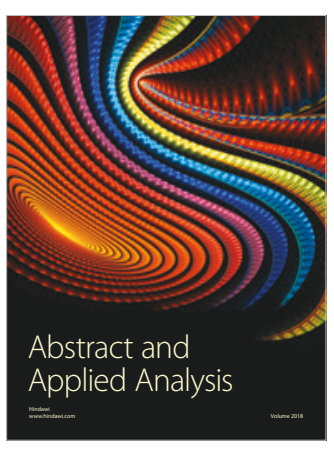

The Scientific

World Journal

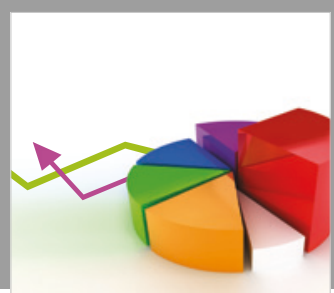

Journal of

Probability and Statistics
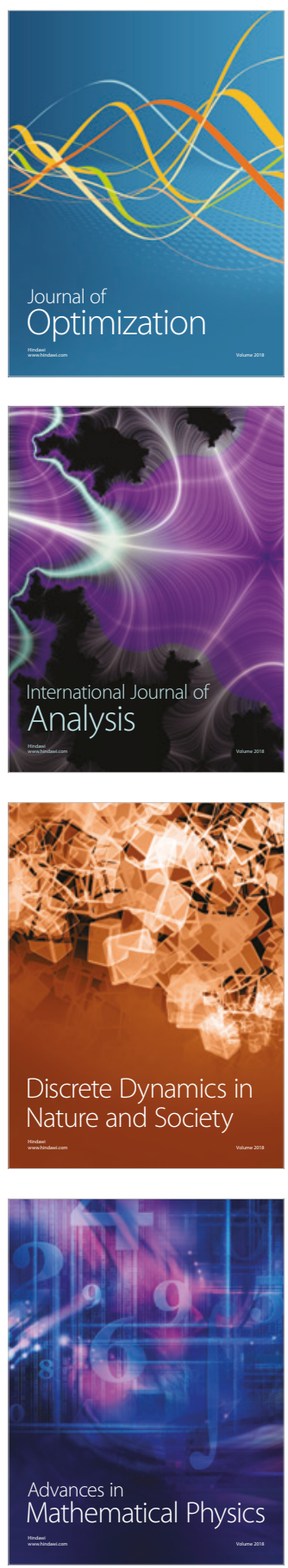